\title{
Exposure to a protein- and tryptophan-deficient diet results in neophilia
}

\author{
STEPHEN F. DAVIS, SCOTT A. BAILEY, and ANN M. THOMPSON \\ Emporia State University, Emporia, Kansas
}

\begin{abstract}
Three experiments were conducted to evaluate the conditioned-taste-aversion account of neophilia. The basic procedure in these experiments involved exposing rats to a protein- and tryptophan-deficient diet of corn grits prior to preference testing. In Experiment 1, the deficient animals consumed more of a novel peppermint flavor than did the normally raised animals. The results of Experiment 2 indicated that the differences shown in Experiment 1 were not attributable to the physiological state present at the time of preference testing. In Experiment 3, the animals selectively avoided a specific flavor that had been associated with the deficient diet. These data are supportive of the taste-aversion account of neophilia.
\end{abstract}

A common paradigm for studying taste-aversion learning (e.g., Garcia, Hankins, \& Rusiniak, 1974; Kalat, 1974) involves (1) exposing experimental subjects to an unfamiliar taste (e.g., saccharin), (2) subjecting them to some form of internal malaise, and (3) measuring their subsequent willingness to consume that taste. Animals administered this routine typically display strong aversions to the novel flavor.

Paradoxically, the taste-aversion paradigm also has been employed to explain neophilia, the preference for a novel flavor or food. For example, Rodgers and Rozin (1966) studied response patterns to new foods in animals raised on a diet deficient in thiamine (vitamin B1). The results clearly demonstrated that thiamine-deficient rats, given an alternative to their familiar, deficient diet, invariably preferred a novel food. The conditioned-taste-aversion account of neophilia (Rozin \& Rodgers, 1967) proposes that animals associate the aversive consequences of consuming a deficient diet with any flavor present during this present state. This conditioned aversion acts to decrease future intake of the particular flavor(s) that were paired with dietary malaise. Therefore, when confronted with this flavor and a second novel flavor, subjects will prefer the novel flavor. This "neophilia" is contrary to the typical reluctance shown by rats to ingest novel foods (i.e., neophobia).

The present studies were conducted to more clearly delineate the conditions under which neophilia can be produced. A second purpose was to evaluate the adequacy of the conditioned-taste-aversion explanation of this phenomenon.

Address correspondence to S. F. Davis, Emporia State University, Psychology Department, 1200 Commercial, Emporia, KS 66801-5087.

\section{EXPERIMENT 1}

The purpose of Experiment 1 was to determine if neophilia would result from exposure to a tryptophan- and protein-deficient diet of corn grits. The conditioned-tasteaversion account of neophilia (Rodgers \& Rozin, 1966) posits that any malaise or discomfort associated with the experimental diet should result in the formation of a conditioned taste aversion to the flavor(s) associated with that diet. When this flavor is then subsequently available, it will be avoided, even if this means ingesting a concomitantly available novel food or fluid. Compared with controls raised on a normal diet of laboratory chow, animals exposed to the grits diet should therefore display stronger neophilia. The occurrence and the duration of neophilia were evaluated through the administration of two daily preference tests following dietary exposure.

\section{Method}

Subjects. Two litters $(n=10$ each) of Holtzman-derived rat pups, born and raised in the Emporia State University vivarium, were subjects.

Apparatus. All testing took place in the home cage. Fluid consumption, calculated to the nearest $.50 \mathrm{ml}$, was measured in $50-\mathrm{ml}$ polypropylene centrifuge tubes fitted with spill-resistant sippers.

Procedure. At birth, the two litters were combined and the pups were randomly assigned to 1 of the 2 dams. The number of males and females was equated as far as possible within each reconstituted litter. The pups remained with their respective dam until weaning (21 days). At weaning, one half of each litter was assigned to the deficient-diet condition (Group Grits), and the remaining animals were assigned to the normal/control condition (Group Normal).

At weaning, each animal was placed in a single, suspended wire-mesh cage and was allowed free access to its designated diet for the remainder of the experiment. The animals in Group Grits received a soupy mixture of Quaker Instant Grits and warm tap water; Group Normal animals received Purina Laboratory Chow. Water was constantly available for both groups. Weight data were obtained for each animal at 30 , 60 , and 90 days of age.

At 90 days of age, all the animals were placed on a fluid-deprivation schedule that permitted them $15 \mathrm{~min}$ of access to water daily. During this 3-day deprivation period, a daily fluid consumption baseline was recorded for each subject. 
A 15-min, two-bottle preference test that pitted peppermint versus water was administered on each of the 2 days that immediately followed the consumption baseline period. The peppermint flavor was prepared by mixing $1.50 \%(\mathrm{v} / \mathrm{v})$ McCormick's Pure Peppermint Extract in tap water.

\section{Results}

Due to the death of 1 Grits animal during the course of the experiment, an unweighted means analysis of variance (ANOVA) was employed for all omnibus $F$ tests. An analysis of the weight data yielded significance for the groups $[F(1,17)=11.24, p<.01]$ and days $[F(2,34)$ $=17.87, p<.01]$ effects. Subsequent Newman-Keuls tests indicated that the Normal animals weighed significantly $(p<.01)$ more than the Grits animals, and that the Normal animals gained a significant $(p<.01)$ amount of weight between the respective weighings. These weight data corroborate previous reports (Becker, Davis, Grover, \& Erickson, 1989, 1990; Remley, Armstrong, Gilman, \& Mercer, 1980) that demonstrate that grits-exposed animals weigh significantly less than their normally reared counterparts.

Because a preliminary analysis of total fluid consumption failed to yield a significant effect, the data for male and female subjects within each group were pooled for further analysis. Prior to analysis, preference test data were converted to consumption ratios by dividing peppermint intake by total (peppermint + water) intake. Thus, ratios above .50 reflect peppermint preferences, and ratios below .50 reflect peppermint aversions.

Figure 1 depicts group mean consumption ratios for the 2 days of preference testing. An ANOVA of the consumption data yielded significance for the days $[F(1,17)=$ $6.75, p=.017]$ and groups $\times$ days $[F(1,17)=6.04, p=$ $.023]$ effects. Reflecting the occurrence of neophilia, subsequent Newman-Keuls tests indicated that the consumption ratios of the Normal animals were significantly $(p<$ .05 ) lower than those of the Grits animals on Day 1. By Day 2, however, the two groups did not differ reliably.

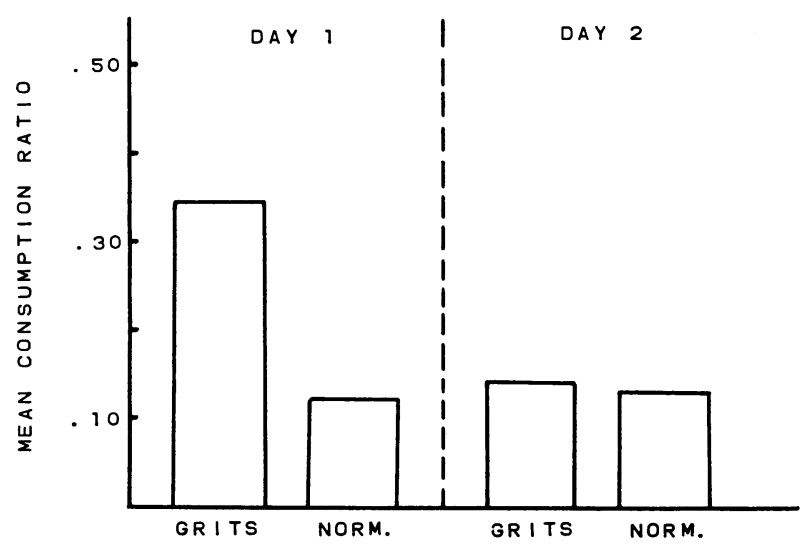

Figure 1. Group mean consumption ratios (peppermint/peppermint + water) for Groups Grits and Normal for the 2 days of preference testing in Experiment 1.

\section{Discussion}

The results of Experiment 1 indicate that, compared with the animals fed a normal diet, those maintained on the protein- and tryptophandeficient grits diet displayed a preference for the novel peppermint flavor (i.e., neophilia). The taste-aversion explanation for this finding assumes that the Grits animals had associated the taste of water, which was consumed during dietary exposure, with the malaise engendered by the deficient diet. Therefore, when confronted with this aversive fluid on the test, along with a novel peppermint flavor, the animals were more willing to sample the peppermint. The finding that the consumption ratios of the two groups did not differ reliably on Preference Test 2 suggests that the aversion to water was weak, probably because it was familiar.

\section{EXPERIMENT 2}

As noted, increased intake of the novel peppermint flavor by the Grits animals in Experiment 1 supports the interpretation that the Grits animals had established an aversion to the fluid consumed during dietary deficiency (i.e., water). Despite this positive outcome, it is noteworthy that the differential dietary conditions imposed at the beginning of the experiment continued throughout the testing phase, so the Grits animals were tested under the continuing effects of the deficient diet, whereas the Normal animals were tested under the effects of the regular diet. One wonders if the difference in physiological state experienced during testing had some influence upon the display of neophilia. Experiment 2 was designed to address this question.

\section{Method}

Subjects. Two litters of Holtzman-derived rats ( $n s=14$ and 15), born and raised in the Emporia State University vivarium, served as subjects. At birth, the litters were culled to 14 animals each and were then combined and reconstituted, as in Experiment 1. The number of males and females was equated as far as possible within each reconstituted litter.

Apparatus. All testing took place in the home cage. Recording of fluid consumption was accomplished as in Experiment 1.

Procedure. At weaning (21 days), one half of each litter was assigned to the deficient-diet condition (Group Grits), and the remaining animals were assigned to the normal/control condition (Group Normal). Each animal was placed in a single, suspended wire-mesh cage.

Coincident with group formation, the animals in Group Grits were placed on the grits diet. To avoid the substantial amount of wasted food encountered in Experiment 1, oven-dried corn grits cakes were used in Experiment 2. These grits cakes were prepared by mixing $100 \mathrm{ml}$ of tap water with $45 \mathrm{~g}$ of Quaker Instant Grits. This mixture was spread on a cookie sheet, baked at $250^{\circ}$ for $2 \mathrm{~h}$, and was then removed and cut into $6.0-\mathrm{cm}$ squares. This period of dietary restriction (Phase 1) remained in place for $\mathbf{4 5}$ days. During this time, Group Normal had free access to the normal diet of Purina Laboratory Chow. Water was freely available to all the animals during this period.

A 45-day recovery period (Phase 2) immediately followed dietary restriction. During recovery, all the subjects received free access to the normal diet and a solution of normal $(.09 \%)$ saline.

Phase 3 (26 days) immediately followed recovery. During Phase 3, one half of the subjects in each group were randomly placed on the grits diet, and the remaining animals in each group received the normal diet. All the subjects received free access to saline during Phase 3. During the final 5 days of Phase 3 , the fluid-deprivation, consumption baseline, and preference test sequence of Experiment 1 were implemented. Thus, one half of the Normal animals (Group Normal-Def) and one half of the Grits animals (Group Grits-Def) received preference testing under the influence of the deficient (Def) grits diet. The remaining Normal animals (Group Normal-Nor) and remaining Grits animals (Group GritsNor) were tested under the influence of the normal diet. 


\section{Results and Discussion}

An analysis of the weight data at the end of Phase 1 indicated that the Normal animals weighed significantly more than the Grits animals $[F(1,26)=8.18, p<.01]$. Reflecting recovery due to the presentation of the normal diet to the Grits animals, the mean weights of the Grits and Normal animals did not differ at the end of Phase 2 $[F(1,26)=2.29, p>.10]$. These data corroborate the Becker et al. (1989) results, which indicate that placing deficient animals on a normal diet reverses the weight differential.

One-way ANOVAs of the weight and consumption data on the final day of consumption baseline did not yield reliable differences among the four subgroups [weight, $F(3,24)$ $=1.56, \mathrm{p}>.10 ;$ consumption, $F(3,24)=1.79, \mathrm{p}>$ $.10]$. Thus, the groups were deemed comparable at the inception of preference testing.

Group mean consumption ratios (peppermint/peppermint + water) for the four subgroups for the two preference tests of Phase 3 are shown in Figure 2. A split-plot factorial ANOVA, incorporating Phase 1 diet (grits vs. normal) and Phase 3 diet (grits vs. normal) as betweensubjects factors and preference tests as a within-subjects factor, yielded significance for the Phase 1 diet factor $[F(3,24)=5.32, p<.01]$. The main effects of testing diet and days, as well as the interactions, failed to achieve significance. The significantly higher consumption ratios displayed by the two Grits subgroups demonstrate the occurrence of neophilia in these animals and corroborate the basic results of Experiment 1. It is noteworthy that, unlike Experiment 1, neophilia was present on the second preference test of Experiment 2 . This is likely due to the greater familiarity of the water in Experiment 1 . During recovery in Experiment 2, all animals drank saline rather than water.

Because the two Grits subgroups (Grits-Def and GritsNor) and the two Normal subgroups (Normal-Def and

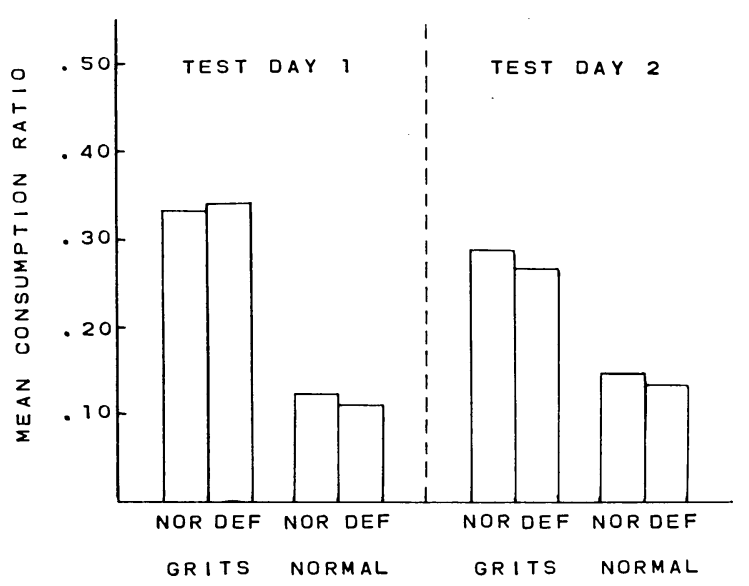

Figure 2. Group mean consumption ratios (peppermint/peppermint + water) for the Grits animals tested under the normal diet (Group Grits-Nor) and the deficient diet (Group Grits-Def), and for the Normal animals tested under the normal diet (Group Normal-Nor) and the deficient diet (Group Normal-Def) in Experiment 2.
Normal-Nor) performed in an indistinguishable manner, it can be concluded that the dietary condition imposed at the time of testing was not an important determinant of neophilia. These results lend additional support to a conditioned-taste-aversion account of this phenomenon.

\section{EXPERIMENT 3}

Even though the results of Experiments 1 and 2 reflect animals' preference for a novel flavor when exposed to a deficient diet, they do not completely substantiate the taste-aversion hypothesis. Perhaps the results of Experiments 1 and 2 are limited to the unique combination of the specific malaise created by the grits diet and the novel peppermint flavor. A stronger, more generalizable case for the taste-aversion explanation could be made if it had been shown that neophilia was not specific to the use of water as the conditioned aversive fluid and peppermint as the novel taste. For example, subjects exposed to saccharin during a period of dietary deficiency would be expected to drink more novel water during a subsequent water versus saccharin preference test. Conversely, those exposed to water during dietary deficiency would be expected to consume more novel saccharin than water. Experiment 3 sought to test these predictions.

\section{Method}

Subjects. Three litters of Holtzman-derived rats, born and raised in the Emporia State University vivarium, served as subjects. At birth, the litters were randomly culled to 12 animals each. The litters were subsequently combined and reconstituted, as in Experiments 1 and 2.

Apparatus. All testing took place in the home cage. The centrifuge tubes and sippers used in Experiments 1 and 2 were also used in Experiment 3.

Procedure. At weaning, each animal was randomly assigned to one of two ( $n=18$ each) groups-WAT (water) and SAC (saccharin)and placed in an individual cage. The distribution of male and female pups was equated as much as possible between the two groups.

All the subjects were maintained on the diet of oven-dried grits cakes for the $\mathbf{4 5}$ days that immediately followed weaning. The groups differed in terms of fluid availability during this 45-day period; Group WAT had free access to plain tap water, and Group SAC had free access to a $.15 \%$ solution $(w / v)$ of sodium saccharin.

Twenty-four hours after the completion of diet exposure, each animal was deprived of fluid for $24 \mathrm{~h}$. At the completion of fluid deprivation, the first 15-min, two-bottle preference test (saccharin vs. water) was conducted. Preference Test 2 was conducted 24 h later.

\section{Results}

An analysis of the weight $[F(1,34)=1.44, p>.10]$ and fluid consumption $[F(1,34)=1.35, p>.25]$ data gathered on the final day of dietary deficiency failed to yield significant between-group effects. Thus, the groups were deemed to be equivalent on these two measures at the start of preference testing.

The preference test data were converted to consumption ratios (see Experiments 1 and 2), and are plotted as group means in Figure 3. An ANOVA performed on these data yielded significant groups $[F(1,34)=30.96, p<$ $.001]$ and groups $\times$ days $[F(1,34)=14.69, p<.001]$ effects. Newman-Keuls tests indicated that Group SAC had significantly $(p<.01)$ lower consumption ratios than did Group WAT on both days of preference testing. Fur- 


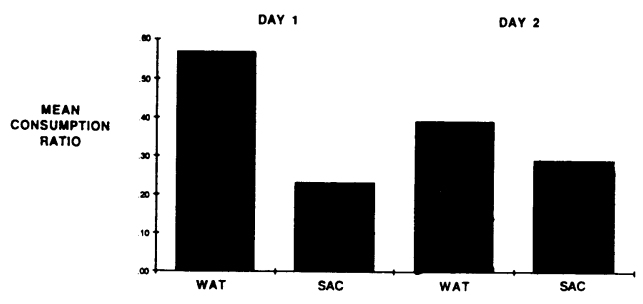

Figure 3. Group mean consumption ratios (saccharin/saccharin + water) for Groups WAT and SAC on Preference Test Days 1 and 2 in Experiment 3.

ther, the consumption ratios of Group WAT were significantly $(p<.01)$ higher on Preference Test 1 than on Preference Test 2.

\section{Discussion}

Because the novel flavor was preferred by both groups during preference testing, these results support the learned-taste-aversion hypothesis. It is clear that, in the presence of the fluid previously paired with the grits diet, consumption of the alternative novel flavor was preferred. When water was associated with grits, the animals in Group WAT preferred to consume saccharin; the converse reaction was evidenced by Group SAC. The results are consistent with the conceptual analysis of neophilia previously offered by Rodgers and Rozin (1966) and Rozin and Rodgers (1967). Although the natural tendency for rats is to avoid novel foods or fluids, when confronted with a choice between an aversive and a novel edible, the novel alternative will be preferred.

It is also noteworthy that neophilia was still present on Day 2 of preference testing in Experiment 3. As in Experiment 2, the persistence of this reaction is likely due to the establishment of a relatively strong aversion to the fluid consumed during deficiency and during testing.

\section{GENERAL DISCUSSION}

Three experiments were conducted to investigate the occurrence of neophilia and to test the conditioned-taste-aversion account of this phenomenon. All three experiments demonstrate the occurrence of neophilia following exposure to a protein- and tryptophan-deficient diet of corn grits. These data complement those of Rodgers and Rozin (1966) and Rozin and Rodgers (1967), which show the development of neophilia following exposure to a vitamin-deficient diet.

In Experiment 1, rats that were exposed to a grits diet subsequently displayed a greater preference for a novel peppermint flavor than did those maintained on a normal diet. In Experiment 2, we demonstrated that the display of neophilia was not influenced by the physiological state (deficient diet vs. normal diet) experienced at the time of preference testing. In Experiment 3, each of two groups of animals had a specific flavor (water or saccharin) associated with the deficient grits diet. Subsequent two-bottle preference tests indicated that each group consumed significantly more of the novel flavor than the flavor that was paired with the deficient diet.

The general implications of these experiments for the establishment of food preferences are worth considering. It is clear that rats have a natural tendency to consume novel foods or flavors with considerable reluctance, a phenomenon known as neophobia (e.g., Barnett, 1963; Mitchell, Kirschbaum, \& Perry, 1975; Richter, 1953; Rozin, 1968; Rzoska, 1953). Neophobia has the obvious adaptive value of reducing the amount of unfamiliar, and hence potentially poisonous, edibles consumed by an animal. This tendency reduces the likelihood of ingesting a lethal amount of a toxic food. However, an animal that survives an encounter with a toxic food or fluid will likely form an aversion to this substance. Consequently, ingestion of this aversive substance should be low under most circumstances. The present data indicate that even novel edibles-substances ordinarily consumed with reluctance-will be preferred to food or fluid that is consumed in conjunction with malaise. This results in a situational preference for novel food, or an apparent neophilia. In the present experiments, the fluid consumed during tryptophan deficiency was likely to be associated with the malaise experienced in this deficient state. When these fluids were introduced during testing, preferences for the alternative, novel fluid were observed. Altogether, these results indicate that the reaction to novel edibles is importantly related to the status of other food or fluids available in the same situation.

\section{REFERENCES}

BARnett, S. A. (1963). The rat: A study in behavior. Chicago: Aldine. Becker, A. H., Davis, S. F., Grover., C. A., \& Erickson, C. A. (1989). The effects of a tryptophan- and protein-deficient diet upon growth in rats. Bulletin of the Psychonomic Society, 27, 345-347.

Becker, A. H., Davis, S. F., Grover, C. A., \& Erickson, C. A. (1990). Effects of a protein- and tryptophan-deficient diet upon complex maze performance. Bulletin of the Psychonomic Society, 28, 126-128.

Garcia, J., Hankins., W. G., \& Rusiniak, K. W. (1974). Behavioral regulation of the milieu interne in man and rat. Science, 185, 824-831.

KalaT, J. W. (1974). Taste salience depends on novelty, not concentration, in taste-aversion learning in the rat. Journal of Comparative \& Physiological Psychology, 86, 47-50.

Mitchell, D., Kirschbaum, E. H., \& Perry, R. L. (1975). Effects of neophobia and habituation on the poison-induced avoidance of exteroceptive stimuli in the rat. Journal of Experimental Psychology: Animal Behavior Processes, 1, 47-55.

Remley, N. R., Armstrong, D. R., Gilman, D. P., \& Mercer, L. F., JR. (1980). Effects of early protein malnutrition on learning in the rat. Bulletin of the Psychonomic Society, 16, 377-379.

RICHTER, C. P. (1953). Experimentally produced behavior reactions to food poisoning in wild and domesticated rats. Annals of the New York Academy of Sciences, 56, 225-239.

RodGers, W., \& Rozin, P. (1966). Novel food preferences in thiamindeficient rats. Journal of Comparative \& Physiological Psychology, 61, 1-4.

Rozin, P. (1968). Specific aversions and neophobia resulting from vitamin deficiency or poisoning in half-wild and domestic rats. Journal of Comparative \& Physiological Psychology, 66, 82-88.

RozIN, P., \& RodGERs, W. (1967). Novel-diet preferences in vitamindeficient rats recovered from vitamin deficiencies. Journal of Comparative \& Physiological Psychology, 63, 421-428.

RzosKA, J. (1953). Bait shyness: A study in rat behavior. British Journal of Rat Behaviour, 1, 128-135.

(Manuscript received January 1, 1993.) 